\title{
DESIGN OF FACE RECOGNITION SYSTEM FOR CANDIDATE VERIFICATION PROCESS PRIOR TO THE EXAMINATION
}

\author{
Geevitha $\mathbf{T}^{\mathbf{1}}$, Hemanth Kumar K $\mathbf{S}^{2}$, Siva Yellampalli ${ }^{3}$ \\ ${ }^{I}$ PG Student, Department of VLSI and Embedded Systems, VTU Extension center,UTI Technologies Limited, \\ Bangalore, India. \\ ${ }^{2}$ Lecturer ,VTU Extension center, UTI Technologies Limited, Bangalore, India. \\ ${ }^{3}$ Principal, United Technologies Ltd, Bangalore
}

\begin{abstract}
A face recognition system which is designed in this project is mainly based on facial geometry measurement. The design work can be divided as two sub-stages, face detection and face recognition. The face detection part of the project will be achieved by the viola jones detector and face recognition part of the design will be done by using artificial neural networks. The developed system can also detect and performs the recognition of multiple faces which are captured in free environment and also recognizes the face from the stored test database. This project is an attempt to work on the problem of human face recognition during candidate verification process prior to the examination. The automated face recognition is implemented using the Matlab technical computing language. The frontal view facial geometry based face recognition system is also expanded and implemented and tested on facial images for different subjects with different poses. The face database of 10 individuals consisting of each 10 different facial images, hence 100 facial images in database to test automated face detection. This project represents the development of a system which can identify the person with the help of a facial features using artificial neural network technique. The features in the image are identified and distance between such identified features are measured. Such measurements are represented as a matrix and this feature matrix is given as input and fed to artificial neural network. The designed system for data base as input could attain 100\% rate of recognition accuracy where as for real time input, rate of recognition accuracy is $96 \%$.
\end{abstract}

Keywords: Facial Geometry Measurement, Face Detection and Face Recognition, Viola Jones Detector, Artificial Neural Network.

\section{INTRODUCTION}

The designed Facial geometry measure based recognition system, first detects the face from query image(test database) or real time acquired image in free environment, then extracts the facial geometry measurements as coordinates which is represented as vector format for input image and matrix format for the images in the database. As the next part of design artificial neural network would recognizes the input images which are acquired in free environment. The designed system is also considered for the illumination compensation by normalizing the light effects of the input image captured in the free environment. The designed system cannot extract the facial geometry measurements if the person is wearing sunglasses and also if person's frontal face is not focused. Also system is designed to perform multi-detection and

multi-recognition. The database being loaded online for competitive and board examination for verification is tedious process for invigilators due to critreas like time, illumination and to avoid cheating the system, by wrong person attempting to take the exam which is an offence. The necessary and requirements of this project is designed and planned to overcome the above problem. The main part of the problem focused is to design and developing facial geometry measurement based face recognition system for the real time application. The designed system is capable enough to detect the faces in the captured images from the free environment, such detected faces will be recognized with the help of artificial neural network. This kind of designed system can be extended as the part of the robotics applications. The project work presents design of facial geometry measure based multidetection and multirecognition with illumination compensation based face recognition system which is simple, costeffective. Since the system does not include complex modules, interfaces, its easy for even nontechnical person to use the system. Also the system is affordable for private institutions since its economical no external costly interfaces instead it includes advanced internal logic design.

\section{RELATED WORK}

Face recognition system is vast and the most emerging technology for most embedded and small smart systems to provide better performance. Ever since the face recognition systems are introduced, there are many much more research works been done on this area. Inorder to develop the efficient and enhanced system as compared to the previous works, literature survey is oriented towards recent and latest works that is been done. The Ma. Christina D. Fernandez[1] 
proposed face recognition system which utilizes viola jones algorithm for detecting the faces from white background and uses artificial neural networks for recognition, with the accuracy of $87.05 \%$.

A comparison of the system[2] that includes the focus on the preprocessing stage. Here, the preprocessing stage involves viola jones face detection, such designed work is compared with respect to gabor, PCA and artificial neural network which can also be used.

The Ashutosh Chandra Bhensle1[3] presents face recognition system which is easy and simple PCA based face recognition system. PCA can increase the recognition accuracy for a face recognition system, in terms of the computational time complexity .. Even this paper is based on viola and jones algorithm for face detection and recognize the face image from multiple sources like still images, webcams and recorded videos. Face detection unit can detect single face as well as multiple faces from different sources.

The Prachi Agarwal[4], has used PCA approach by calculating eigenface values for feature extraction and back propagation neural network for classification This approach reduces the computation load to distinguish an image from the database. Firstly multilevel decomposition using Haar wavelet transform has been implemented on an image to break up it into multilevel sub image bands. After that improved PCA is applied on the subband images for extracting Eigen values.

The Amr El Maghraby[5] and M A Imran[6] has used viola and jones algorithm for detection purpose. The work is oriented towards to detect the face in the complex background with clearly improved Viola - Jones face detection accuracy and decreasing false negative rates. The certain constraints considered for design using eigen face is the various parameters like illumination, different pose, background colour and lighting conditions.

The Raunil Singh[7] has done work on different types of face detection techniques, algorithms have been studied and described and is followed by the changes to be made to develop a new hybrid algorithm for face detection technique, which will overcome the false positive result in face detection and recognition technique. The system designed is based on back propagation neural network with Fuzzy logic, normalise Cross-Correlation, Principle Discriminate analysis.

Optimization towards practical application is very important for face recognition system. The optimization requires comparison of other techniques. Such comparison and optimized work is done by Marian Dorin Pirloaga[8]. It is a Applied research work which targets at obtaining software for real-time recognition of individuals with the most advanced method recognized by Viola Jones. The application does not allow the recognition of the upper face covered or emotional states and implementation of the portable computer terminals and interconnected systems assume that in military terms is difficult.

The Vikas Maheshkara[9] introduces the methodology for extracting facial geometric measurements which involves information regarding direction and multiscale. Also this design can automatically performs the calculations of measurements of facial parts. It is based on characterization and geometric relationship of the facial parts. By characterizing the facial features the design can be built.

The M.M. Fakhir[10] proposed methodology mainly focuses on finding the unique and different dimensions for ears, inorder to frame unique and different features format. The precise technique harvesting dimensions is used to find the distance. This work did not necessitate expensive camera, the system could work for mobile camera. The geometric shape of square is the reference for the parameters to calculate measurements.

\section{SYSTEM WORKFLOW}

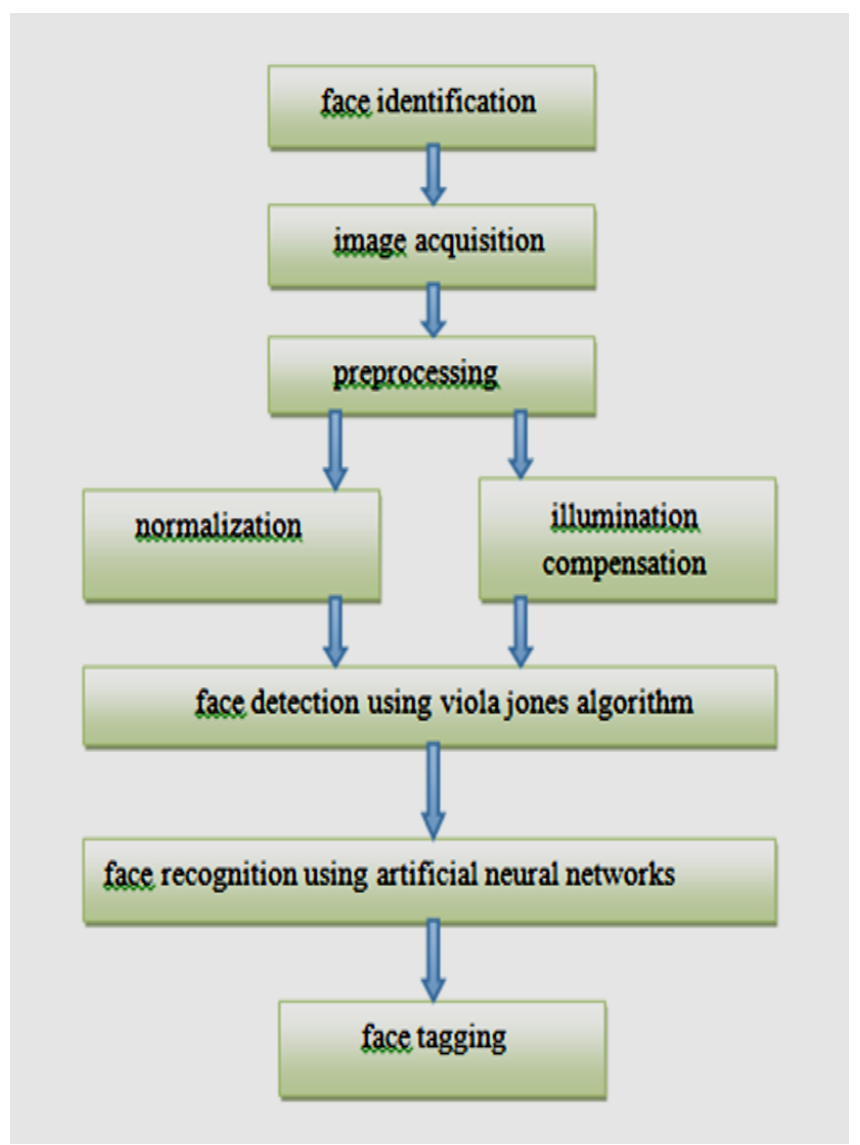

Fig 1. Face recognition system workflow

The system modeling plan is as shown in the figl.which gives the brief idea of the project flow. The system is designed to work for both real time application and standard bio-id database. Image acquisition tool is required for configuring the camera to capture the real time image capture. The captured images are in the format of the free environment, since the verification environment prior to the examination cannot be restricted to the white background.The system design is also considered for 
standard bio-id database which is suitable for real time applications. The database is created using BioID database .The database is formed using 10 different persons of each 10 different poses. The database totally includes 100 images which are captured in free environment.The query image from database or real time captured image through camera is taken as input. Viola jones algorithm is used to detect the face from captured image in free environment, the identified face is cropped and resized. The preprocessing involves the process of resizing the captured image or input image by only detecting the face part in the input image. Such resized image is made to undergo illumination compensation, which will normalizes the input image's colour effect. The illumination compensation process helps to improvise the efficiency by reducing the colour effects. Here Preprocessing is done for better visualization of image, like contrast adjustment which is achieved with the help of illumination compensation. Also viola jones detector identifies the main facial parts in the input face image as a part of face detection process. It will identify the pair of eyes, nose, mouth and represent it as co-ordinates. Proceeding further the facial geometry measurement is done to find 6 parameters and such 6 parameters are trained to artificial neural network to match the target with input and gives the output .Parameters are facial parts geometry measurements. These parameters are calculated and stored as a matrix format, for different persons. such procedure of calculating matrix format is done for all the images which is stored in database and trained to the ANN. The parameters of input image is calculated and trained to ANN as feature vector. The matrix calculated for entire database is given to ANN as target and input image's extracted feature vector as in the form of parameters are given as input to ANN. When the query image is given as test image to ANN, based on the results the right person is identified by artificial neural networks.

\section{EXPERIMENTAL RESULTS}

A. Face detection and preprocessing by viola jones detector for test database input

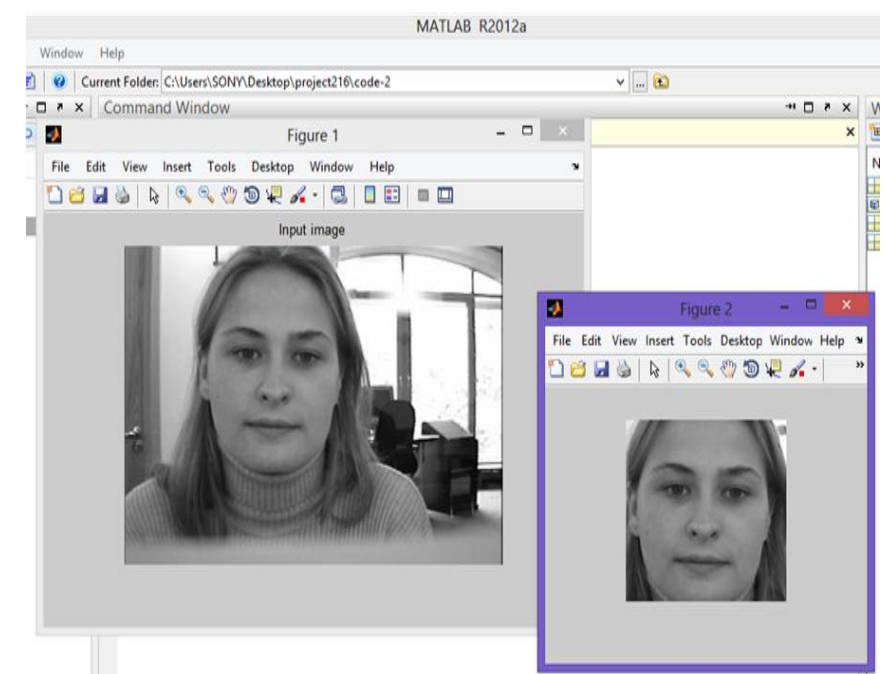

Fig 2. face detection for free environment captured database
B. Facial parts identification by viola jones detector

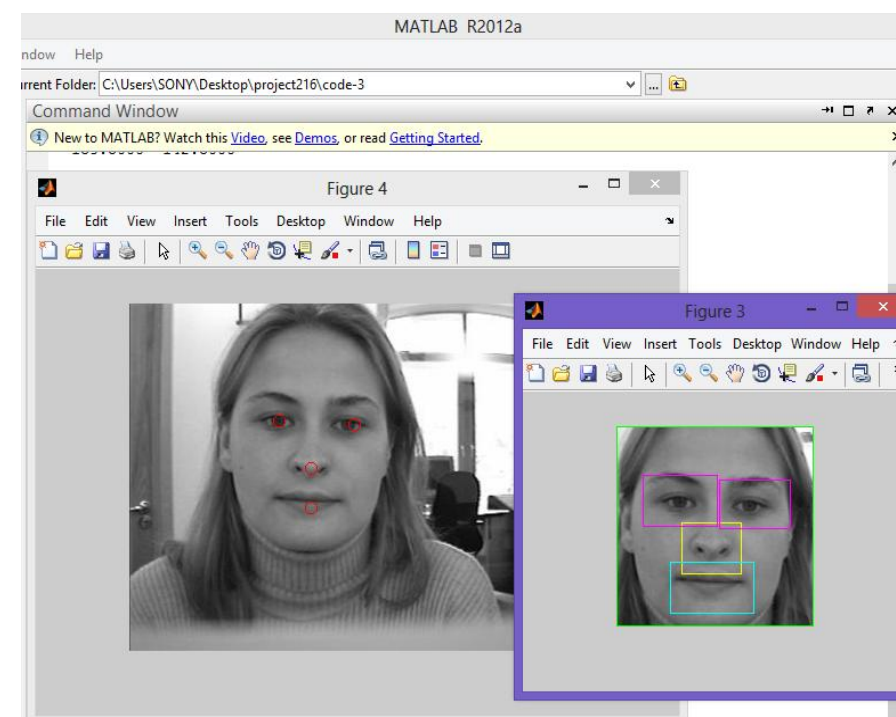

Fig 3. Facial feature identification

C. Face recognition by ANN

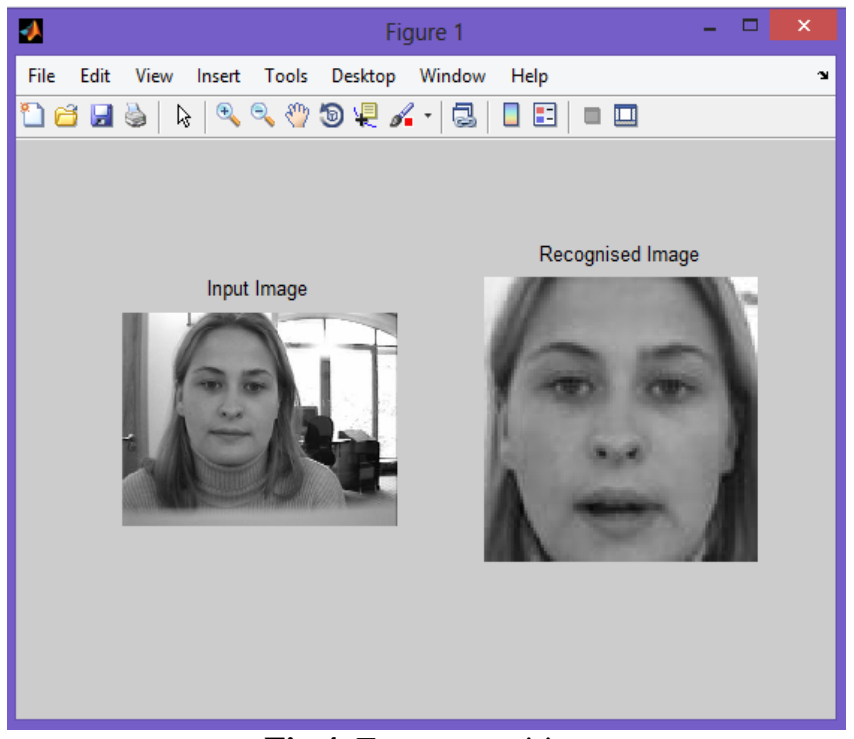

Fig 4. Face recognition

D. Face detection and preprocessing by viola jones detector for real time input

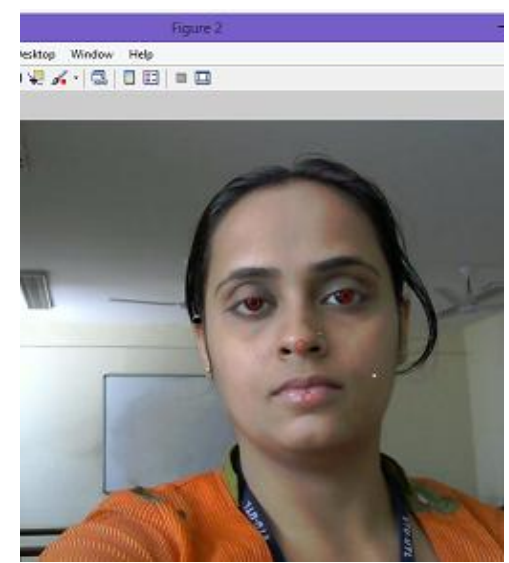

Fig 5. face detection by viola jones detector 
E Face recognition by ANN for real time input

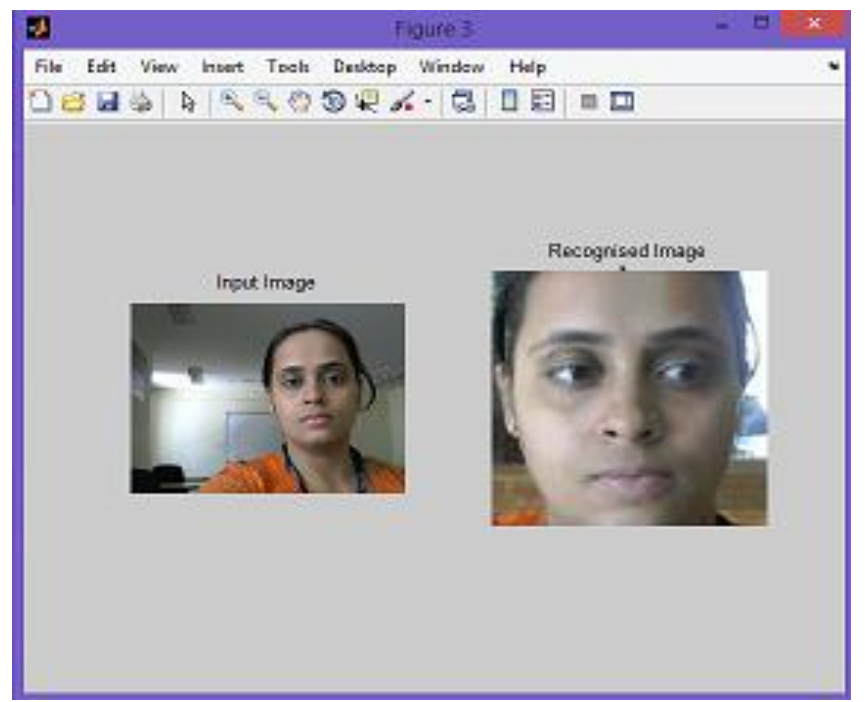

Fig 6. Face recognition by artificial neural network

\section{CONCLUSION}

The design and development of the facerecognition system could identify the person's face in free environment instead of white background.To detect the face from free environment and to identify facial main parts llike eyes, nose, lips the most efficient viola jones detector is used. In this project the unique way of facial geometry measurement technique is used and images to be detected is undergone illumination compensation technique. Also this project is capable of performing multi-detection and multirecognition.The designed system uses artificial neural network to recognize the query image. The database involved in this project is the standard form BIO-ID database which is suitable for real time applications. System recognizes 10 people atleast. The designed system cannot extract the facial geometry measurements if the person is wearing sunglasses and also if person's frontal face is not focused The designed system can be integrated and extended for robotics applications and the methodology implemented can be useful for passport verification by working on aging factor of persons to be verified.

\section{REFERENCES}

[1] Ma. Christina D. Fernandez, Kristina Joyce E. Gob, Aubrey Rose M. Leonidas ,Ron Jason J. Ravara, Argel A. Bandala, Elmer P. Dadios, "Simultaneous Face Detection and Recognition using Viola-Jones Algorithm and Artificial Neural Networks for Identity Verification ", Proc. IEEE Transactions on Computers, 978-1-4799-2027-3/14/\$31.00 @2014 IEEE.

[2] Mohammad Da'san, Amin Alqudah and Olivier Debeir Computer Engineering Department, Hijjawi College, Yarmouk University, Irbid, Jordan, Université libre de Bruxelles, Bruxelles, "Face Detection using Viola and Jones Method and Neural Networks ", IEEE Transactions on Computers, vol. 54, no. 8, pp. 10251040, 2005.
[3] Ashutosh Chandra Bhensle, Rohit Raja “An Efficient Face Recognition using PCA and Euclidean Distance Classification ", In International symposium on mobile computing, Automation, and Test, IJCSMC, Vol. 3, Issue. 6, June 2014, pg.407 -413.

[4] Prachi agarwal, Naveen prakash. "Modular Approach for Face Recognition System using Multilevel Haar Wavelet Transform, Improved PCA and Enhanced Back Propagation Neural Network", International Journal of Computer Applications (0975 - 8887) Volume 75- No.7, August 2013.

[5] Amr EI Maghraby ,Mahmoud Abdalla "Hybrid Face Detection System using Combination of Viola - Jones Method and Skin Detection", International Journal of Computer Applications (0975 - 8887) Volume 71No.6, May 2013.

[6] M A Imran,M S U Miah "Face Recognition using Eigenfaces ", International Journal of Computer Applications (0975 - 8887) Volume 118 - No. 5, May.

[7] Raunil Singh,Kiran Gupta "Face Detection and Recognition - A Review", SSRG International Journal of Computer Science and Engineering (SSRG-IJCSE) EFES April 2015.

[8] Marian,Ciprian “OPTIMIZING FACE RECOGNITION IN IMAGES", Review of the Air Force Academy No 1 (28) 2015.

[9] Vikas Maheshkar,Sunitha Agarwal. "Face recognition using geometric metrics ",2012.

[10] M M Fakhir, W L Woo "Face Recognition Based on Features MeasurementTechnique”,November2014. 